\title{
油浸式电力变压器绝缘故障分析和处理
}

靳文德

青海铜业有限责任公司

DOI:10.32629/hwr.v3i6.2195

[摘 要] 油浸式电力变压器的绝缘主要通过绝缘材料而实现, 是变压器正常作业的基本条件; 此外, 变压器的使用寿命与绝缘 材料的寿命息息相关。电力变压器作为电网构成的最基本元器件之一,变压器的可靠性对于电网的正常运行有着十分重要的 影响。在变压器的绝缘中, 尽管国内外都致力于开发无油变压器, 但是现今最主流的变压器多是油浸式电力变压器, 其依靠油和 纸板来进行变压器绝缘。本文重点探究油浸式电力变压器绝缘故障的判断和处理措施,包括变压器油、渗漏点等处理, 以提高 油浸式电力变压器的处理效率、质量。

[关键词] 油浸式变压器; 绝缘故障; 处理措施

油浸式电力变压器是现今较为主流的变压器, 随着我国 电力建设速度的不断加快, 油浸式电力变压器正在向着大 型、特大型变压器的方向发展。油浸式电力变压器从设计完 成到出厂投入运行, 需要经历层层工序。为确保油浸式电力 变压器到现场后能够正常使用, 需要确保其运输安全。

\section{1 油浸式电力变压器的结构}

1.1 目前容量较大的变压器均为油浸式电力变压器, 其 绕组及铁芯浸泡在绝缘油中, 绕组经绝缘套管引出, 从而与 外电路连接。

1. 2 铁芯和绕组构成了变压器的主体框架, 通常铁芯会 采用厚度为 $3.5^{\sim} 5 \mathrm{~cm}$ 的硅钢片叠装而成; 绕组根据电压大小 可分为高压、中压、低压绕组; 绕组通过绝缘纸缠绕包裹, 绕组匝间, 高中低压绕组之间, 绕组与铁芯及地之间, 均通过 绝缘垫块进行隔离并实现彼此间绝缘。

1. 3变压器油是一种矿物油, 具有良好的绝缘性能。对于 油浸式电力变压器来说, 变压器油的主要作用在于:

1. 3. 1 提高变压器绕组之间及其与铁芯、油箱间的绝缘 性能。

1.3. 2变压器油在一定的高温条件下, 油体温度和流速 会迅速提升, 逐渐形成对流空间, 避免变压器铁芯和绕组因 集中发热而造成短路现象。

\section{2 油浸式电力变压器常见绝缘故障}

相关运行经验表明, 油浸式电力变压器常见的绝缘故障 主要有以下几类: 内部局部放电; 绕组变形导致绝缘垫块脱 落或绝缘纸破损; 变压器内部进水或受潮; 绝缘纸或垫块等 固体绝缘老化等。

\section{3 故障综合诊断方法}

3. 1 内部局部放电。为检验变压器内部是否发生局部放 电故障, 可采取油色谱分析的方法进行判断。通过油色谱分 析的方法, 能初步判断变压器内部是否存在低能放电、高能 放电、还是存在过热故障。再结合绕组直流电阻、变比试验、 绝缘电阻测试等试验项目, 基本能对故障发生的原因及部位 进行判断。
3. 2 绕组变形故障。在变压器发生出口近区短路的情况 下, 如果变压器本身的阻抗短路能力不强, 极有可能发生绕 组变形故障, 进而影响其内部绝缘。为此, 在变压器发生出口 短路后, 应对变压器采用的检测方法包括: 油色谱分析; 短 路阻抗试验; 空载电流试验等, 以此判断变压器是否发生绕 组变形故障。

3. 3变压器绝缘受潮。为检验变压器绝缘是否出现受潮 现象, 则应对变压器的绝缘电阻、介质损耗、极化指数、油 中微量水分含量、变压器油色谱分析等进行初步检测和判断, 必要时可开展绝缘特征试验以及绝缘纸的含水量检测试验。

3. 4 固体绝缘老化。通常情况下, 为判断变压器是否出现 固体绝缘老化现象时, 应采用色谱分析法对变压器油中的一 氧化碳、二氧化碳的含量变化等进行分析, 同时开展油中糠 醛含量、绝缘纸、油酸值等方面的检测工作。

3. 5 溶解气体的在线监测技术。目前, 油中溶解气体分析 作为一种较为成熟的技术, 能判断出油浸式变压器内部发生 的绝大部分故障。通过对变压器加装溶解气体的在线监测装 置, 定期取样实现实时监测变压器内部绝缘油所分解的特征 气体含量 $(\mathrm{H} 2 、 \mathrm{CO} 、 \mathrm{CO} 2 、 \mathrm{CH} 4 、 \mathrm{C} 2 \mathrm{H} 4 、 \mathrm{C} 2 \mathrm{H} 6 、 \mathrm{C} 2 \mathrm{H} 2)$, 可以实 现对变压器故障进行实时而有效的诊断。以便当在运变压器 出现内部故障时, 及时采取有效的处理措施, 提高故障的处 理效率, 降低设备及电网事故的发生风险。

3.6 露点法。露点法主要用于检测变压器纸绝缘的含水 量。对于充气运输的变压器, 可通过此方法检测变压器是否 在运输过程中受潮。同时, 对怀疑受潮的变压器, 也可通过取 纸样的方法, 判断受潮影响程度。此方法的原理是: 当变压 器内部气体达到一定条件后, 并在气体和油纸中水分平衡的 情况下, 根据气体露点测量结果推断纸绝缘的含水量。若变 压器内部充气时间较短, 可通过多次测量的方式判断气体和 油纸中水分是否处在一个平衡状态, 若在 $12 \mathrm{~h}$ 内测量值保持 不变, 即可视为这二者达到一种平衡状态。

\section{4 浸式电力变压器绝缘故障处理措施}

4. 1处理变压器油的措施。针对变压器绝缘受潮故障, 
可采用热油循环的方法进行现场处理, 所采取的故障处理措 施为: 关闭瓦斯继电器油箱侧阀门和散热器阀门, 将变压器 本体的油位控制在低于箱顶 $30 \mathrm{~cm}$ 以下。在油处理过程中, 滤 油机压力表、真空压力表均控制在 $0.3 \mathrm{MPa} 、 0.09 \mathrm{MPa}$, 出口油 温以 $75^{\circ} \mathrm{C}$ 为宜, 做好热油循环处理。当本体油温升至 $60^{\circ} \mathrm{C}$, 方可计时, 维持热油循环 $36 \mathrm{~h}$ 后, 将从设备内部脱气 $2.5 \mathrm{~h} 。$ 静 止24h后, 对变压器低压绕组绝缘电阻、吸收比、泄露电流进 行测试。

4. 2 处理渗漏点。若是变压器检查显示存在渗漏点, 需做 好针对性的处理工作, 具体如下:

4. 2. 1 将变压器油全部排至油罐, 对高压侧德尔孔门渗 漏点进行补焊, 更换损坏的密封垫。

4.2.2 将导电装置拧紧、螺母压紧, 确保密封垫固定牢靠 后, 变压器油内氢气进行加热过滤、真空脱气处理, 实现有效 消除。

4.2.3完成真空脱水脱气处理后, 开展热油循环处理工 作, 将变压器油内氢气、一氧化碳、二氧化碳彻底去除。

4. 2. 4真空注油, 若是油色谱显示正常、相关试验达标后, 表明故障已处理完成, 可结束操作。

4. 3其它绝缘故障处理。若经检测, 发现变压器存在绕组 变形, 绝缘纸破损, 绝缘垫块大范围松动脱落等类型的绝缘故 障, 需将绕组重新绕制。因现场机具人员以及环境等原因, 往 往难以控制施工工艺及施工质量, 故此类故障不推荐进行现 场处理, 对此类故障, 建议返厂, 由变压器厂家进行专门处理。

\section{5 油浸式变压器日常运行维护措施}

在油浸式变压器运行过程中, 绝缘故障是影响其稳定运 行的主要因素之一。为了进一步减少或避免绝缘故障对变压 器运行造成的影响, 需要做好以下几点措施:
5. 1安装、运行和检修维护部门应在具体工作中保持严 谨、认证的工作态度, 严格按照相关操作流程和规范, 减少故 障发生率。

5.2 结合变压器设备的运行时间、运行状态等确定例行 试验检修周期, 通过例行试验结果, 对变压器设备的状况进 行诊断, 若状态诊断结果表明变压器存在异常, 应及时采取 措施进行处理和控制。

5.3 有效控制变压器的正常运行。加大对变压器日常运 行的监管力度, 包括变压器顶层油温、套管顶部油温和气体 继电器等, 确保在监测过程中就能发现变压器设备存在的安 全隐患或故障。

5. 4严格控制变压器大修吊罩等工作中, 绕组和铁芯在空 气中的暴露程度, 确保真空干燥、真空注油操作能够严格按照 相关规范进行, 消除绝缘件附带的水分, 保证其完全干燥。

\section{6 结语}

总而言之, 为准确分析和判断油浸式电力变压器绝缘故 障, 需要采用多种试验及诊断技术, 综合分析, 综合判断。同 时, 在变压器的日常运维中, 还应注意变压器日常运行状态, 例行试验数据, 在线诊断数据等, 通过对各类数据的综合分 析和把控, 及时发现并处理其内部绝缘的早期隐患, 进而保 障油浸式变压器的安全稳定运行。

[参考文献]

[1]邓世建, 张宽,胡继普,等.油浸式电力变压器故障与其 本体外表面温度关系分析[J].工矿自动化,2017,43(6):25-31.

[2] 魏云冰,王东晖,韩立峰,等。一种基于MIA的油浸式变压器放 电性故障定位新方法[J].电力系统保护与控制,2015,43(21):41-47.

[3]蒲丽娟,刘念,刘航宇, 等. 基于油浸变压器故障数据的 IEC 三比值统计分析 [J]. 陕西电力,2015,43(05):41-44+49. 